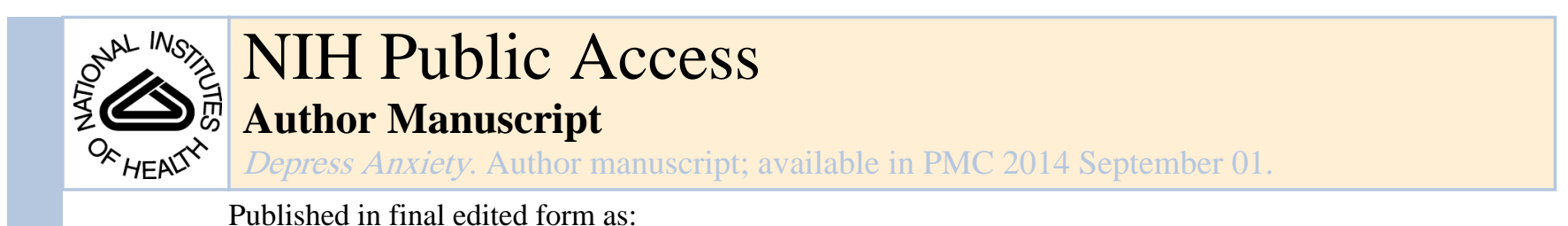

Depress Anxiety. 2013 September ; 30(9): 787-791. doi:10.1002/da.22171.

\title{
Biomarkers in Pediatric Depression
}

\author{
Uma Rao \\ Center for Molecular and Behavioral Neuroscience, and the Department of Psychiatry and \\ Behavioral Sciences, Meharry Medical College, Nashville, TN, USA; The Department of \\ Psychiatry and Kennedy Center, Vanderbilt University, Nashville, TN, USA
}

\begin{abstract}
Depression is a leading cause of morbidity and mortality in youngsters. Elevated risk for the disorder begins in the early teens and continues to rise in a linear fashion throughout adolescence, with lifetime rates estimated to range from $15 \%$ to $25 \%$ by late adolescence. Numerous studies have documented that early depressive episodes persist or recur into adult life along with ongoing psychosocial difficulties. A better understanding of the etiology and pathophysiology of pediatric depression will be helpful in the development and implementation of more effective primary and secondary preventive strategies, thereby allowing such youth to achieve their full potential as adults.
\end{abstract}

There is a general consensus that depression results from complex interactions between multiple genetic and environmental factors. Endophenotypes or biomarkers help target the underlying mechanisms. The biomarkers also can be used to strengthen classifications of clinical phenotypes, or to differentiate possible biological subtypes that may, in turn, have different clinical or treatment profiles. In order to better characterize the potential biomarkers associated with pediatric depression, a summary of the literature on adult depression will be provided; in contrast to the wealth of information available in adults, empirical data in youngsters are limited by relatively modest sample sizes in far fewer studies.

\section{Biomarkers in Adult Depression}

While there is no clear single biomarker associated with depression, there is mounting evidence of contributing factors, including sleep, neuroendocrine, inflammatory, metabolic, neurotrophic and neural networks. ${ }^{[1-5]}$

\section{Sleep}

There are a number of reasons to consider the regulation of sleep as an essential component for understanding the pathophysiology and treatment of depression. ${ }^{[2,6]}$ There is a significant overlap in the control of sleep and mood regulation. Sleep complaints are commonly associated with depression and form an essential criterion of the diagnosis. Developmental influence(s) on the rates of depression and maturational changes in sleep regulation also imply a close connection between depressive disorders and sleep regulation. Depression and sleep "abnormalities" often co-segregate among family members. Certain sleep markers have been detected in healthy individuals at high familial risk for depression and they were associated with the development of depression during prospective follow-up. Sleep alterations often persist beyond the clinical episode of depression and increase the

Corresponding Author: Uma Rao, M.D., Center for Molecular and Behavioral Neuroscience, Meharry Medical College, 1005 Dr. D.B. Todd Jr. Boulevard, Nashvile, TN 37208, USA. TEL: 615-327-6875; FAX: 615-327-6144; urao@mmc.edu.

Financial Disclosures

The author has no financial conflicts of interest. 
vulnerability to relapse or recurrence. Sleep changes also predict treatment response, and many antidepressant treatments impact sleep.

Although no single sleep marker is specifically associated with depression, a constellation of sleep changes has been observed. The most reliable sleep macroarchitectural changes associated with major depression include sleep continuity disturbances (e.g., delayed sleep onset, and decreased sleep efficiency), earlier onset of rapid eye movement (REM) sleep, increased REM activity and REM density, increased amount of REM sleep, and diminished slow-wave sleep (SWS) ${ }^{[7]}$ Specifically, individuals experiencing major depression and those in remission exhibit increased REM density and shortened SWS, as do persons at high familial risk. Therefore, this combination of sleep features may represent a genetic biomarker of depression. Further, SWS appears to be even shorter during remission following a depressive episode, suggesting its role as both a genetic marker as well as a biological scar of the disorder.

\section{Neuroendocrine System(s)}

There has been considerable interest in the hypothalamic-pituitary-adrenal (HPA) system, consistent with the hypothesis that depression is linked to altered responses to stress. ${ }^{[4,5]}$ Depression is associated with higher basal corticotropin-releasing hormone and cortisol secretion. ${ }^{[8]}$ Increased HPA activity in depression is due, in part, to altered feedback inhibition of the HPA axis by endogenous glucocorticoids. It has been proposed that elevated cortisol in patients with depression is a compensatory mechanism in response to decreased glucocorticoid receptor function and expression in the brain. Increased cortisol secretion frequently persists during remission and increases the risk for relapse/recurrence of depression. ${ }^{[3]} \mathrm{HPA}$-axis hyperactivity also was observed in unaffected individuals at familial risk for depression and predicted the onset of depression, suggesting that it may be a genetic vulnerability marker of depression. ${ }^{[3,9]}$

\section{Inflammatory Markers}

Evidence suggests that inflammation may have a critical role in the pathophysiology of depression. ${ }^{[1,3,4]}$ Clinical studies demonstrated that patients with depression have elevated blood/serum levels of inflammatory markers, including pro-inflammatory cytokines. Cytokine activation produces sickness behaviors, which share features with depression. Moreover, chronic stress exposure produces changes in immune function that may influence the pathophysiology of depression. Consistent with these findings, inhibiting proinflammatory cytokine signaling in patients with inflammatory disorders, as well as in patients with depression, improves mood and facilitates antidepressant treatment response. Although most of the data on the association between depression and inflammatory markers are cross-sectional, several lines of research indicate that the link between inflammation and depression is likely bidirectional. ${ }^{[3]}$

\section{Metabolic Function}

Evidence indicates a bidirectional relationship between depression and metabolic dysregulation. ${ }^{[3,4]}$ Circulating hormones, such as leptin and ghrelin, relay information regarding peripheral energy homeostatic levels to the brain. Leptin receptors are expressed in the limbic system, and leptin also has been shown to affect hippocampal and cortical structures through its actions on neurogenesis, axon growth, synaptogensis and dendritic morphology. Low levels of leptin are associated with depressive behaviors, and chronic stress exposure decreases serum leptin. Consistent with these results, acute leptin administration produces antidepressant effects and increases the expression of brain-derived neurotrophic factor (BDNF) in the hippocampus. By contrast, chronic stress exposure increases serum ghrelin levels. Calorie restriction produces antidepressant effects which are 
mediated by ghrelin. ${ }^{[4]}$ Hence, leptin and ghrelin may serve as putative biomarkers for depression in general, or in depressed patients with altered metabolic function.

\section{Neurotrophic Factors}

Chronic stress exposure, which can precipitate or exacerbate depressive episodes, alters the expression of neurotrophic (growth) factors. By contrast, antidepressant treatment enhances trophic factor expression and neuroplasticity. ${ }^{[4]}$ Among the various neurotrophic factors, BDNF has been best investigated in both preclinical and clinical studies. BDNF is transcribed at relatively high levels and expressed in several peripheral tissues, and plasma/ serum BDNF levels might be derived from these tissues as well as the brain. Although the functional significance of plasma/serum BDNF is not known, recent studies in animals suggest that peripheral growth factors (including BDNF) can enter the brain and produce both behavioral and cellular responses. Several studies have reported reduced plasma/serum BDNF levels in patients with depression, and that antidepressant treatment normalizes these levels. ${ }^{[4,5,10]}$

\section{Neural Networks}

A growing body of unimodal structural and functional neuroimaging studies found alterations in frontolimbic and frontostriatal circuits in depression. ${ }^{[5,11]} \mathrm{A}$ few investigations utilizing multi-modal methods have suggested that structural alterations are related to functional changes, while others reported functional alterations in the absence of structural changes. ${ }^{[11]}$ Alterations in neural structure/function also have been associated with the treatment of depression (as predictors of clinical response and/or as changes in response to antidepressant treatments). ${ }^{[12]}$

\section{Biomarkers in Pediatric Depression}

Although there are far fewer studies with modest sample sizes in pediatric depression, many of the biomarkers described in adult depression also have been observed in pediatric depression. For example, reduced REM latency and REM density and hypercortisolemia not only have been observed during a depressive episode, but also in unaffected youth at high familial risk for depression, and these biomarkers predicted the subsequent onset of depression. ${ }^{[9]} \mathrm{A}$ bidirectional relationship between depression and inflammatory markers was observed during prospective follow-up in unaffected adolescents who were at high-risk for depression based on familial or cognitive vulnerability, particularly in those who experienced childhood adversity. ${ }^{[13]}$ Depression was associated with inflammatory and metabolic markers in youth with diabetes. ${ }^{[14]}$ Reduced neurotrophic factors (including BDNF) have been reported in pediatric depression. ${ }^{[15]}$ Alterations in frontolimbic and frontostriatal circuits also have been observed in structural and functional neuroimaging studies of early-onset depression, with some studies reporting these changes in unaffected youth at high familial risk. ${ }^{[16]}$

The advantage of studying pediatric samples is that we can identify premorbid (vulnerability) markers in high-risk populations so that they can be targeted for preventive interventions and reduce the economic and social burden associated with depression. Even individuals with the disorder are in the early course of illness and will be ideal for identifying markers associated with treatment-resistance or poor prognosis. Also, early-onset illness is highly familial and is associated with a chronic recurrent course, possibly representing a unique subtype that could be targeted for identifying genetic and epigenetic correlates. ${ }^{[17]}$ 


\section{Integrative Summary of Biomarkers in Depression}

Given that depression is a heterogeneous disorder, it is unlikely that any given biomarker has a high degree of sensitivity and specificity to make it clinically useful. Hence, the development of panels that aim to profile a diverse array of biomarkers to provide coverage of multiple biological abnormalities that contribute to the heterogeneity of depression, and its response to treatment, is a potentially promising approach. Consistent with this theme, a combination of biomarkers seem to aggregate more consistently in some patients but not in others. Such clustering may be more closely related to the etiology of a depression subtype and, in turn, could lead to more effective, etiologically-based treatments for subgroups of patients. ${ }^{[3,4]}$

Although it appears that the above-described biomarkers are diverse, they are inter-related. For example, sleep regulation is not only linked to mood, but also to cognitive, endocrine, immune and metabolic functions. Inflammatory markers, including cytokines, regulate neuroendocrine function, and reciprocally glucocorticoids have inhibitory effects on inflammation. Both HPA and immune systems affect metabolic function, and vice versa. Activation of inflammatory pathways and reduced leptin and glucocorticoid receptor function within the brain are believed to contribute to decreased neurotrophic support and altered glutamate release/reuptake, as well as oxidative stress, leading to excitotoxicity and loss of glial elements, consistent with neuropathologic findings that characterize depression.

Genetic variants have a moderating influence on the association between biomarkers and depression phenotypes and/or treatment response. For example in different clinical studies, the Val66Met BDNF polymorphism ("met-allele") was associated with higher depression severity, lower serum BDNF levels, smaller hippocampal volume or abnormal hippocampal activity during an episodic memory task, but better response to antidepressant treatment, compared to the Val-allele. ${ }^{[5]}$ An emerging literature suggests that stress exposure induces epigenetic mechanisms, such as histone modifications and deoxyribonucleic acid (DNA) methylation, and alters the expression of many of these biomarkers. Therefore, tracking genetic variants and epigenetic changes might compliment biomarker panels..$^{[1,4,5]}$

\section{A Multi-dimensional Biological Model of Depression}

Based on the multi-dimensional concept, Schneider et al. proposed an exemplary biological model of depression (see Figure 1). ${ }^{[5]}$ This model consists of three levels: A neuronal network level (assessed with structural and functional neuroimaging methods as well as with neurophysiological methods), a molecular systems level (assessed using proteomic, lipidomic and transcriptomic approaches) and a genetic/epigenetic level. Additionally, environmental factors are considered as an important factor within this model, as they interact with neurobiological systems on all three levels. Various constellations of gene-gene and gene-environment interactions can lead to imbalances within and across these three major pathways, culminating in depressive disorder. On the other hand, such interactions also could have buffering effects and promote resilience in at-risk individuals.

Consistent with this theme, a recent study identified 26 candidate blood transcriptomic markers from genome-wide analysis of two animal models, representing the genetic and environmental (stress-related), etiology of depression. ${ }^{[18]}$ They applied these markers in a sample of adolescents with depression $(n=14)$ and controls with no disorder $(n=14)$. A panel of 11 blood markers differentiated depressed and control groups. Additionally, a separate but partially overlapping panel of 18 transcripts distinguished depressed youth with or without comorbid anxiety. These data should be replicated in larger samples before these markers can be applied clinically. 
A multi-dimensional approach, as described in Figure 1, requires large sample sizes to obtain adequate power to define severity and identifiable subtypes of depression. Results obtained from the biomarker panels will need to be reproducible and standardized such that clear associations between these bio-signatures and clinical subtypes are readily apparent. Some approaches, such as neuroimaging and sleep electroencephalography, are not practical or cost-effective in routine clinical practice. Instead, ambulatory measures such as nearinfrared spectroscopy (NIRS) might be more suitable. ${ }^{[19]}$

\section{Clinical Application of Biomarkers for Depression}

Ridge Diagnostics developed a blood-based diagnostic test, Major Depressive Disorder Score (MDDScore). This multi-analyte immunoassay detects four major biological pathways: inflammation, HPA axis, metabolic and eurochemical pathways. A mathematical algorithm provides an MDDScore which predicts the probability of depression with high sensitivity and specificity. ${ }^{[20]}$ The MDDScore is yet to be compared across laboratories and tested on a large population of patients with a variety of psychiatric disorders before the test is standardized and available for use in routine clinical practice. The Food and Drug Administration has approved genotyping tests for common variants of drug metabolism genes (e.g. cytochrome P450). These tests help the physician to select an appropriate antidepressant drug for a given patient, as differences in clearance, half-life and peak blood concentrations are controlled by genetic variability in drug metabolism. They are especially helpful in alerting to possible adverse effects and to optimize dose. ${ }^{[1]}$

\section{Conclusions}

Currently, there is no clear biomarker profile associated with depression for clinical use. However, based on a combination of preclinical and clinical studies, a biochemical profile has emerged that can be tested in clinical populations. In the meantime, existing information on genetic biomarkers and the availability of genotyping tests concerning genetic control of drug metabolism and associated toxicity can help the physician in selecting a safe antidepressant for a given patient. A truly personalized medicine approach for depression will be achieved only when the biomarkers assays are widely available and can be considered to be cost-effective diagnostic tests. Research directed toward the discovery of biomarkers associated with depression and its response to treatment is of the utmost importance in this endeavor.

\section{Acknowledgments}

This work was supported, in part, by grants the National Institutes of Health (R01 MH068391, G12 RR003032/ MD007586, UL1 RR024975/TR000445 and U54 RR026140/MD007593), and by the Endowed Chair in Brain and Behavior Research at Meharry Medical College.

\section{References}

1. Miller DB, O'Callaghan JP. Personalized medicine in major depressive disorder -- opportunities and pitfalls. Metabolism. 2013; 62(Suppl 1):S34-9. [PubMed: 23021040]

2. Palagini L, Baglioni C, Ciapparelli A, et al. REM sleep dysregulation in depression: State of the art. Sleep Med Rev. 2013

3. Penninx BW, Milaneschi Y, Lamers F, Vogelzangs N. Understanding the somatic consequences of depression: biological mechanisms and the role of depression symptom profile. BMC Med. 2013; 11:129. [PubMed: 23672628]

4. Schmidt HD, Shelton RC, Duman RS. Functional biomarkers of depression: diagnosis, treatment, and pathophysiology. Neuropsychopharmacology. 2011; 36(12):2375-94. [PubMed: 21814182] 
5. Schneider B, Prvulovic D, Oertel-Knochel V, et al. Biomarkers for major depression and its delineation from neurodegenerative disorders. Prog Neurobiol. 2011; 95(4):703-17. [PubMed: 21854829]

6. Rao U. Sleep Disturbances in Pediatric Depression. Asian J Psychiatr. 2011; 4(4):234-247. [PubMed: 22287998]

7. Pillai V, Kalmbach DA, Ciesla JA. A meta-analysis of electroencephalographic sleep in depression: evidence for genetic biomarkers. Biol Psychiatry. 2011; 70(10):912-9. [PubMed: 21937023]

8. Stetler C, Miller GE. Depression and hypothalamic-pituitary-adrenal activation: a quantitative summary of four decades of research. Psychosom Med. 2011; 73(2):114-26. [PubMed: 21257974]

9. Rao U, Hammen CL, Poland RE. Risk markers for depression in adolescents: sleep and HPA measures. Neuropsychopharmacology. 2009; 34(8):1936-45. [PubMed: 19262465]

10. Hashimoto K. Brain-derived neurotrophic factor as a biomarker for mood disorders: an historical overview and future directions. Psychiatry Clin Neurosci. 2010; 64(4):341-57. [PubMed: 20653908]

11. Hamilton JP, Chen MC, Gotlib IH. Neural systems approaches to understanding major depressive disorder: an intrinsic functional organization perspective. Neurobiol Dis. 2013; 52:4-11. [PubMed: 23477309]

12. Fu CH, Steiner H, Costafreda SG. Predictive neural biomarkers of clinical response in depression: a meta-analysis of functional and structural neuroimaging studies of pharmacological and psychological therapies. Neurobiol Dis. 2013; 52:75-83. [PubMed: 22659303]

13. Miller GE, Cole SW. Clustering of depression and inflammation in adolescents previously exposed to childhood adversity. Biol Psychiatry. 2012; 72(1):34-40. [PubMed: 22494534]

14. Hood KK, Lawrence JM, Anderson A, et al. Metabolic and inflammatory links to depression in youth with diabetes. Diabetes Care. 2012; 35(12):2443-6. [PubMed: 23033243]

15. Pallavi P, Sagar R, Mehta M, et al. Serum neurotrophic factors in adolescent depression: Gender difference and correlation with clinical severity. J Affect Disord. 2013

16. Hulvershorn LA, Cullen K, Anand A. Toward dysfunctional connectivity: a review of neuroimaging findings in pediatric major depressive disorder. Brain Imaging Behav. 2011; 5(4): 307-28. [PubMed: 21901425]

17. Talati A, Weissman MM, Hamilton SP. Using the high-risk family design to identify biomarkers for major depression. Philos Trans R Soc Lond B Biol Sci. 2013; 368(1615):20120129. [PubMed: 23440463]

18. Pajer K, Andrus BM, Gardner W, et al. Discovery of blood transcriptomic markers for depression in animal models and pilot validation in subjects with early-onset major depression. Transl Psychiatry. 2012; 2:e101. [PubMed: 22832901]

19. Takizawa R, Fukuda M, Kawasaki S, et al. Neuroimaging-aided differential diagnosis of the depressive state. Neuroimage. 2013

20. Souslova T, Marple TC, Spiekerman AM, Mohammad AA. Personalized medicine in Alzheimer's disease and depression. Contemp Clin Trials. 2013

Depress Anxiety. Author manuscript; available in PMC 2014 September 01. 


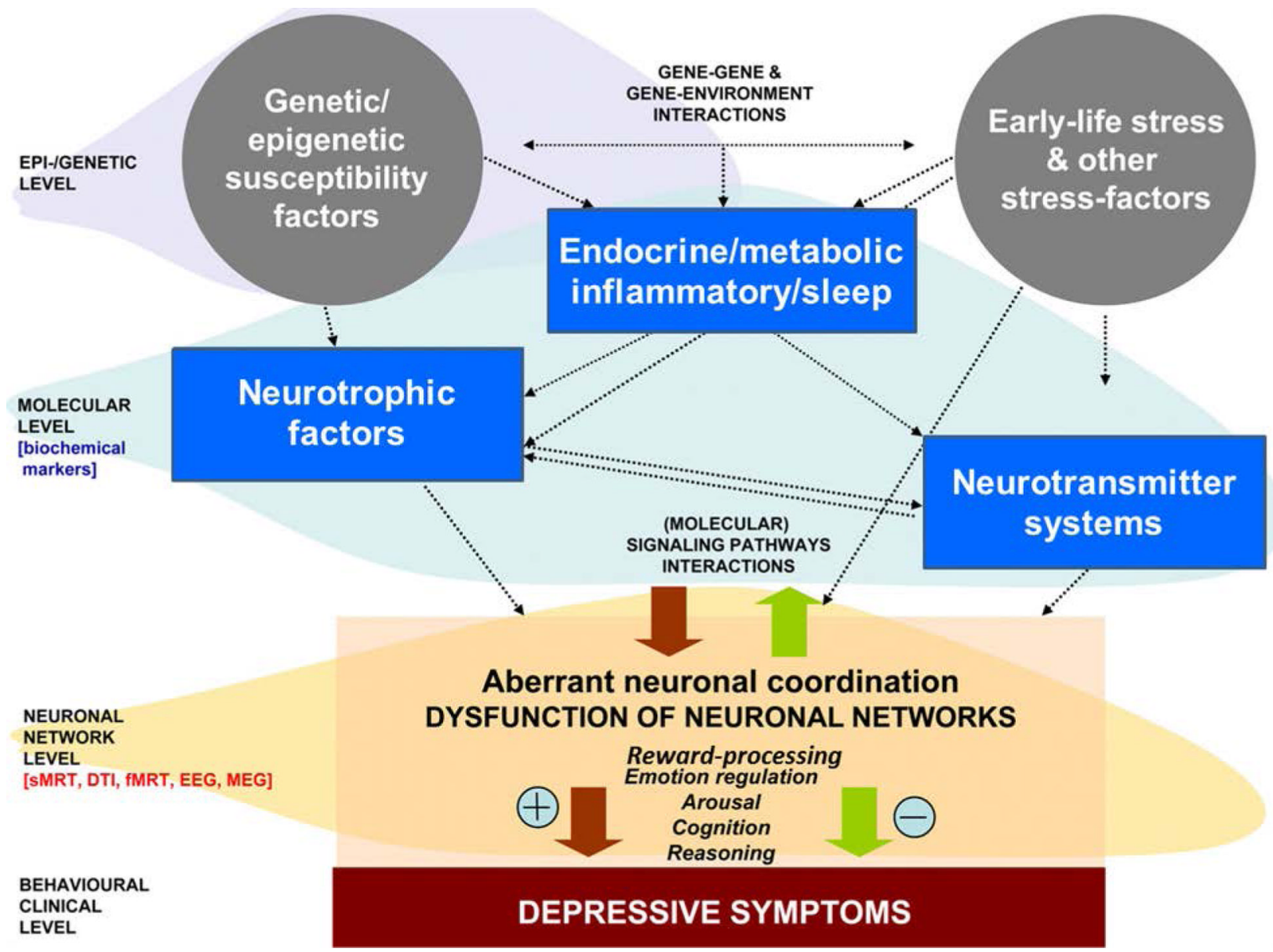

Figure 1.

A Biological Model of Depression (adapted from B. Schneider Riemann et al., Progress in Neurobiology, 2011;95:703-717). (Schneider 2011) 